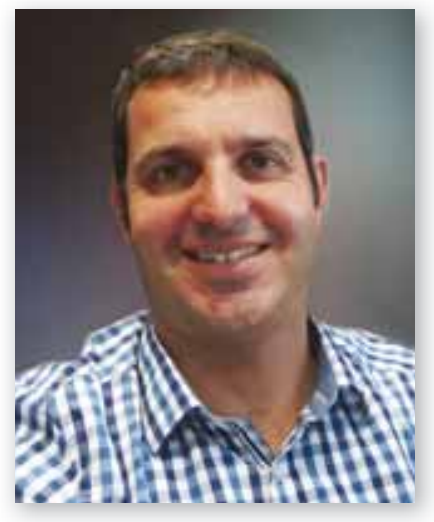

\author{
Guest editorial \\ Prof Jacques Janson \\ Head: Division of Cardiothoracic surgery, University of Stellenbosch and \\ Tygerberg Hospital, Tygerberg, South Africa
}

\title{
Embracing new techniques and new technologies in cardiac surgery - is it being done responsibly and safely?
}

\begin{abstract}
Cardiac surgeons have been pioneers since the inception of this surgical field. Sixty years ago the development of the heart-lung machine paved the way for cardiac surgery. Since then many new innovations have been introduced, including artificial heart valves, coronary artery bypass grafting, cardiac transplantation, minimally invasive techniques, transcutaneous heart valves and mechanical assist devices. This has required parallel development and advances in medical imaging, such as angiography, echocardiography, CT scanning and MRI providing accurate preoperative diagnosis and optimal planning of surgical procedures. We have come a long way since the serendipitous discovery of coronary angiography by Mason Sones in 1957 when he mistakenly injected the right coronary artery with dye during an angiographic examination of a young man with aortic stenosis, thus paving the way for percutaneous coronary interventions and coronary artery bypass surgery. ${ }^{(1)}$
\end{abstract}

Cardiac surgery is rapidly evolving and today's cardiothoracic surgeon needs to acquire expertise, not only in the standard surgical procedures, but also in minimally invasive and transcatheter valve therapies. The field of mechanical circulatory support and the various device options are also increasing, and although it is very expensive technology, it will lead to a greater demand for specialised surgeons who are capable of implanting these devices and who are experienced in the appropriateness of their use.

How do we incorporate new therapies safely into our practice without compromising on patient outcome? A surgeon who wishes to start a new procedure or learn a new technique must first assess whether the new procedure is safe and reproducible and whether there are enough patients in his or her referral base who will need the procedure. Discussions with referring cardiologists are helpful in identifying suitable patients who will benefit from a new procedure and whether it is viable. Not only must the surgeon venture into a new technique in close collaboration with the referring cardiologists, but all the members of the heart team (including the anesthetists, nurses and technologists) must embrace the new skills that are required for the procedure. Unlike the introduction of a new pharmaceutical drug which can be readily defined and standardised, the introduction of a new surgical procedure consists of several components that can affect the outcome. (2) These components include surgeon characteristics such as surgical skill, decision making, preferences and level of experience as well as the skills of the other team members, such as the anesthetists, nurses and technologists. Pre- and postoperative management vary between centres and is affected by infrastructure, staffing and local policies which can 
affect the outcome of a surgical procedure. Educating the whole team (cardiologist, radiologist, anesthetist, scrub nurse, technologist and intensive care unit staff) is essential, so that everyone is comfortable with the steps and their role in the new procedure.

Visiting high volume centres of excellence for specific procedures can shorten the learning curve by identifying indications, contra-indications and certain pitfalls of a procedure. A visiting surgeon, experienced in the procedure, also facilitates the safe initiation of a new procedure. As with any surgical procedure, it must be done with attention to detail and technical precision. A self-assessment and analysis of results is important once the procedure is started, to see whether it is beneficial and to find method for improvement.

Our heart team faced many of these challenges when a transcatheter aortic valve implantation (TAVI) programme was initiated. The heart team concept had already been established in our institution where we have weekly meetings between cardiologists and surgeons to discuss patients for coronary artery bypass grafting, or percutaneous therapy. It was quite a natural process to also discuss potential TAVI patients in this way. The surgeon and cardiologist visited Leipzig, a high volume centre in TAVI, to gain experience and did the required training courses. Imaging specialists from the ranks of both the radiologists and cardiologists became involved with pre-operative imaging to define aortic and femoral vessel anatomy and disease and to define the aortic annulus anatomy and size. The anesthesiology team, nursing staff and technologists were also educated in the steps of the new procedure. An experienced proctor visited the unit to help with the first procedures which shortened the learning curve and enabled us to perform the procedure safely. The TAVI registry in South Africa is well maintained and is a valuable tool to monitor the results of the new procedure. The remaining problems which we face include the cost of the new devices and the resulting limitation on the amount of TAVIs which can be done, an important requirement to develop and maintain expertise with the procedure.

It will become more difficult in future for a single cardiac surgeon to treat all the cardiac pathologies with the latest techniques or technology. The value of large centres lies in that they can have a surgeon with expertise in a specific field, such as valve repair, coronary artery surgery, TAVI, mechanical assist devices and transplantation. Ideally, we should aim towards having larger cardiac centres in South Africa where surgeons can develop a specific interest and expertise. Currently, we have many small cardiac units with I or 2 surgeons. It is unreasonable to expect that these surgeons perform a minimally invasive procedure, for example, if they have not been trained in this skill. It is then better to perform the best and safest procedure which the surgeon is comfortable with for a good patient outcome. 
Guest editorial, Jacques Janson
In Leipzig, a large centre which does between 350 - 450 minimally invasive mitral valve repairs per year, they found the average learning curve for a surgeon showed a turning point to lower complications following 75 - 125 cases.(3) They also showed a clear tendency towards lower complications if the surgeon performed minimally invasive mitral valve surgery at least twice per week. These numbers are impossible to obtain in a small heart centre which does 50 - 150 total cardiac cases per year.

We have to adapt our cardiothoracic surgical training programmes to ensure that surgeons who qualify are sufficiently equipped and specialised to thrive in their clinical practice. It will not be possible to train each surgeon in all the latest technological skills, but the skillsets that the surgeons learn must be relevant to their clinical practice. It will be valuable for surgeons to learn catheter skills as transcatheter heart valve technology and aortic stenting increases. Newly qualified surgeons should be encouraged to hone their skills in a specific field by doing a fellowship in a high volume centre of excellence.

Cardiac surgery is evolving, and while this is exciting, it expands the need for continuing medical education and the relearning of certain skill sets. The responsibility for ensuring the safety, efficacy and value of new technology or techniques lies with the surgeon. ${ }^{(4)}$ This challenge provides the opportunity for intellectual, scientific and academic fulfillment while we ensure that each patient receives the best procedure for his or her heart disease.

\section{REFERENCES}

I. Cosgrove, D. M. (200I). Developing new technology. The Journal of thoracic and cardiovascular surgery, I2I (4), S29-S3I.

2. Ergina PL, Cook JA, Blazeby JM, et al., Balliol Collaboration. Challenges in evaluating surgical innovation. The Lancet. 2009;374(9695): 1097-104.

3. Holzhey DM, Seeburger J, Misfeld M, et al. Learning minimally-invasive mitral valve surgery: a cumulative sum sequential probability analysis of 3895 operations from a single high volume center. Circulation. 20 I3:CIRCULATIONAHA-II 3.

4. Whitson BA, Lima B. Surgery for acquired cardiac disease: An evolving paradigm with a promising future. The Journal of thoracic and cardiovascular surgery. 2016;151(6):1466-9. 